Cristina Pérez-López ${ }^{1}$

M. ${ }^{a}$ Dolores López-Franco ${ }^{1,2, *}$

Inés María Comino-Sanz ${ }^{1,2}$

Pedro L. Pancorbo-Hidalgo $0^{1,2}$

1. Grupo de investigación Enfermería e innovación en cuidados de salud. Universidad de Jaén. Jaén. España.

2. Departamento de Enfermería. Facultad de Ciencias de la Salud. Universidad de Jaén. Jaén. España.

*Autor para correspondencia.

Correo electrónico: mlfranco@ujaen.es (M. Dolores López Franco).

Recibido el 2 de agosto de 2019; aceptado el 31 de agosto de 2019.

\section{Actitud hacia la prevención de lesiones por presión en estudiantes de enfermería: cuestionario APuP}

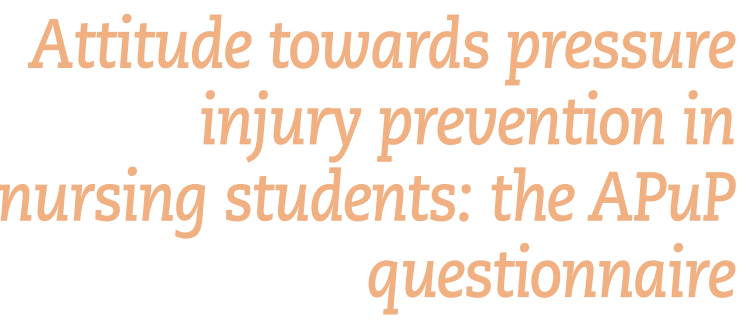

\section{RESUMEN}

Objetivos: Conocer la actitud hacia la prevención de las lesiones por presión que tienen los estudiantes y las estudiantes del Grado en Enfermería.

Objetivos secundarios: a) realizar la valoración psicométrica de la versión española del cuestionario Attitudes towards Pressure ulcer Prevention

(APuP) (Actitudes hacia la prevención de las UPP) en población de estudiantes y $b$ ) identificar los factores formativos asociados con la actitud.

Métodos: Estudio observacional transversal, llevado a cabo en la Universidad de Jaén mediante un cuestionario online en estudiantes de los 4 cursos del Grado en Enfermería. Se utilizó el análisis de Rasch para establecer las propiedades psicométricas del cuestionario. Se calculó la puntuación de actitud y su asociación con variables formativas. Para estimar el tamaño

del efecto se usó la diferencia estandarizada de medias ( $d$ de Cohen).

Resultados: Se analizaron un total de 188 cuestionarios válidos. La puntuación media obtenida en el cuestionario APuP fue 22,60 (44,23\% del máximo). El cuestionario presenta una buena consistencia global $(\alpha=0,74)$ y los índices de ajuste de los ítems fueron buenos. Se encontró una actitud menos positiva en estudiantes que habían realizado prácticas clínicas $(p<0,0001)$ y los de los cursos superiores $(p<0,0001)$. Existe una correlación inversa entre la puntuación de actitud y la puntuación de conocimientos $(\mathrm{r}=-0,46)$. Conclusiones: El cuestionario APuP (versión española) es un instrumento válido y fiable para su uso en estudiantes de enfermería. La actitud hacia la prevención no es positiva en estos estudiantes y se evidencia un posible efecto de la realización de prácticas clínicas y el aumento de conocimientos en una actitud menos favorable.

PALABRAS CLAVE: Lesión por presión, prevención, estudiantes de enfermería, encuestas y cuestionarios, actitudes.
ABSTRACT

Objectives: To explore the attitude towards pressure injury prevention of nursing students. Secondary objectives: a) To establish the psychometric properties of the questionnaire of Attitudes towards the prevention of pressure ulcer (APuP) -Spanish version- in student population, and $b$ ) To identify the educational factors associated with the attitude. Methods: A cross-sectional observational and validation study was carried out with students of the four years of the Bachelor in Nursing in the University of Jaén (Spain) using an online survey. Rasch analysis was used to establish the psychometric properties of the questionnaire. Also, the score of attitude and its association with educational variables were analysed. The standardised median difference (Cohen's d) was used to estimate the effect size. Results: A total of 188 valid questionnaires were analysed. The average score obtained in the APuP questionnaire was 22.60 ( $44.23 \%$ of the maximum). The questionnaire has a good overall internal consistency $(\alpha=0.74)$ and the item fit indices were good. It was found a less positive attitude in students who had done clinical placements $(p<0.0001)$ and those in higher years $(p<0.0001)$. There is an inverse correlation between the attitude score and the knowledge score $(r=-0.46)$. Conclusions: The questionnaire of attitudes towards prevention of pressure ulcers (APuP) - Spanish version - is a valid and reliable instrument to use in nursing students. The attitude towards prevention is not positive in these students and there is some evidence for a possible effect of clinical placements and increased knowledge over a less favourable attitude.

KEYWORDS: Pressure injuries, Prevention, Nursing students, Surveys and questionnaires, Attitude.

\section{$\searrow$ INTRODUCCIÓN}

Las lesiones por presión (LPP) se deben a un daño localizado en la piel y el tejido blando subyacente, generalmente sobre una prominencia ósea, como resultado de la presión o presión más cizalla ${ }^{1,2}$. Estas lesiones suponen un evento adverso en la asistencia sanitaria y tienen una elevada prevalencia en pacientes hospitalizados. En España, según el $5^{\circ}$ Estudio Nacional de Prevalencia realizado por el Grupo Nacional para el Es- 
tudio y Asesoramiento sobre Úlceras por Presión y Heridas Crónicas (GNEAUPP) en 2018, la prevalencia fue del 7\% en adultos hospitalizados $^{3}$ y del 3,3\% en unidades pediátricas ${ }^{4}$. Las LPP pueden causar un gran daño a los pacientes, en forma de dolor, angustia, complicaciones y hospitalización prolongada ${ }^{5,6}$, generando así importantes costos económicos para los proveedores de servicios de salud?

La prevención de las LPP es considerada un importante indicador de calidad de la atención de enfermería. Requiere un enfoque multidisciplinar, aunque las enfermeras deben desempeñar un papel de liderazgo en su prevención en los entornos sanitarios ${ }^{8}$. Las medidas de prevención no solo pueden ser utilizadas para prevenir la aparición de LPP, también pueden disminuir el dolor y las complicaciones que pueden derivarse, la duración de los tratamientos y, por consiguiente, el gasto sanitario?.

La formación de los profesionales y un nivel adecuado de conocimientos ${ }^{10}$ son una parte esencial en el proceso de prevención de las LPP, ya que la falta de conocimientos puede llevar a conceptos erróneos. No obstante, algunos estudios apuntan a que los conocimientos son insuficientes por sí solos, ya que están influidos por las actitudes y por las razones que tienen los profesionales de enfermería para aplicarlos en la práctica del día a día ${ }^{11,12}$. Por tanto, la prevención de las LPP implica no solo adquirir los conocimientos a través de estrategias educativas, sino también promover una actitud positiva hacia este aspecto de la atención al paciente ${ }^{13}$.

Los estudios que analizan los niveles de conocimientos en diferentes países y contextos muestran una gran variabilidad en sus resultados, aunque globalmente indican deficiencias de conocimientos sobre la prevención. No todos los instrumentos publicados presentan datos de fiabilidad y validez psicométrica ${ }^{14}$. Los cuestionarios más utilizados que ofrecen evaluación psicométrica son el Pieper Pressure Ulcer Knowledge (PPKUT) ${ }^{15,16}$, el Pressure Ulcer Knowledge Assessment Tool $\left(\right.$ PUKAT) ${ }^{10,17}$ y el Pressure Injury Prevention Knowledge (PIPK) ${ }^{18}$.

Con respecto a las actitudes de los enfermeros y las enfermeras hacia la prevención de las LPP hay menos estudios. Una reciente revisión de la literatura científica ${ }^{19}$ permite identificar varios instrumentos válidos para medir estas actitudes, como la Escala de actitudes hacia la prevención de las úlceras por presión de Moore y Price ${ }^{20}$ y el cuestionario Attitudes towards Pressure ulcer Prevention (APuP $)^{21-23}$. Los hallazgos de estos estudios indican de forma general una actitud positiva hacia la prevención $^{19}$.

A pesar de que los estudios realizados para conocer la actitud de los estudiantes y las estudiantes de enfermería son menos numerosos que los dirigidos a profesionales de enfermería en activo, la literatura internacional indica que los primeros tienen actitudes apropiadas y positivas para la prevención; sin embargo, sus conocimientos y habilidades son deficientes e inadecuados ${ }^{8,24-28}$. Se espera que los estudiantes lleven a cabo las medidas de prevención de manera efectiva y precisa, pero la forma en que estos realizan las actividades de prevención está relacionada con sus conocimientos y actitudes básicas que reciben en los planes de estudios ${ }^{5,8}$. Por tanto, es importante incluir en los planes de estudios el desarrollo de los conocimientos, habilidades y actitudes necesarios para la prevención de LPP con el objetivo de asegurar que enfermeras y enfermeros recién graduados estén preparados para el entorno clínico ${ }^{26}$, ya que se ha comprobado que el conocimiento deficiente y las actitudes negativas llevan a menor prevención de las LPP en la práctica ${ }^{20,25}$.

Los estudios sobre prevención de LPP enfocados a estudiantes de enfermería en España son muy escasos, por lo que esta investigación se enfocó en medir tanto los conocimientos como la actitud hacia la prevención de las LPP en estudiantes del Grado en Enfermería en una universidad española. Los resultados sobre los conocimientos de los es- tudiantes han sido descritos en otro artículo ${ }^{29}$ y en este se describen los resultados sobre la actitud.

\section{Y OBJETIVOS}

El objetivo principal fue explorar la actitud hacia la prevención de las LPP que tienen los estudiantes y las estudiantes del Grado en Enfermería. Objetivos secundarios fueron: realizar la valoración psicométrica del cuestionario $\mathrm{APuP}$ (versión española) en población de estudiantes e identificar los factores formativos que se asocian con la actitud.

\section{MÉTODOS}

Se realizó un estudio observacional transversal utilizando un formulario online; el periodo de recogida de datos fue entre febrero y marzo 2019.

\section{Participantes y muestra}

La población de estudio fueron estudiantes del Grado en Enfermería de la Universidad de Jaén, invitando a participar a estudiantes matriculados en los cuatro cursos del grado. No se aplicó ningún criterio de exclusión. La muestra final quedó formada por estudiantes que aceptaron completar la encuesta.

\section{Procedimiento de obtención de datos}

Para la recogida de datos se diseñó un formulario online, a través de una plataforma de encuestas online segura (Surveymonkey), garantizando la privacidad de la información. Este constaba de una sección demográfica, y el cuestionario $\mathrm{APuP}^{21}$ en su versión española. Dicho formulario online fue enviado a través de la plataforma de Docencia Virtual (DV), previa explicación del objetivo del estudio y la forma de acceder a la encuesta en una sesión presencial durante una clase. Posteriormente, se enviaron mensajes recordatorios a través de la plataforma para motivarlos a completar el cuestionario.

\section{Variables}

La variable principal fue la actitud hacia la prevención de las LPP medida mediante el cuestionario APuP (versión española) ${ }^{22,30}$. Este cuestionario, en su versión original, consta de 13 ítems agrupados y 5 factores con 4 opciones de respuesta (totalmente en desacuerdo, en desacuerdo, de acuerdo, totalmente de acuerdo, que se puntúan de 1 a 4) y una puntuación máxima de 52 puntos. Antes de calcular la puntuación total es necesario invertir la puntuación de los ítems 3, 4, 5, 7, 8, 10 y 13 (de forma que totalmente en desacuerdo puntúa 4 en vez de 1). Los factores del cuestionario son: factor 1 (competencia personal para prevenir LPP), con los ítems 1, 2, 3; factor 2 (prioridad de la prevención de las LPP), ítems 4, 5 y 6; factor 3 (impacto de las LPP), ítems 7, 8 y 9; factor 4 (responsabilidad en la prevención de las LPP), ítems 10 y 11, y factor 5 (confianza en la eficacia de la prevención), ítems 12 y 13.

Además, se recogieron variables sociodemográficas: edad, sexo, curso más alto matriculado, trabajo previo en el ámbito de la salud, realización de prácticas clínicas, lugar donde han realizado las prácticas, número de periodos de prácticas y asistencia a alguna jornada/congreso sobre LPP en el último año. El cuestionario de Conocimientos sobre la prevención de lesiones por presión (PIPK) en versión española se administró de forma paralela para medir los conocimientos. 


\section{Análisis de los datos}

Para establecer las propiedades psicométricas del cuestionario APuP (versión española) se usó la teoría de respuesta al ítem ${ }^{31}$ mediante un análisis Rasch. El análisis Rasch permite obtener información sobre el rendimiento de los ítems y de las personas de manera independiente $\mathrm{y}$ calcula la puntuación de la variable latente medida (theta), por lo que resulta muy útil en el análisis de cuestionarios que miden rasgos personales como conocimientos y actitudes ${ }^{32}$. Como el cuestionario APuP usa una escala Likert de 4 puntos, se empleó el método de Andrich para análisis de variables policotómicas ${ }^{33}$. Para cada ítem del cuestionario se calculó la puntuación de dificultad y los índices de ajuste al modelo: media cuadrada no ponderada de los residuos estandarizados (UMS, Unweighted Mean Squared) y media cuadrada ponderada de los residuos estandarizados (WMS, Weighted Mean Squared). Para su interpretación se considera que valores de UMS y WMS entre 0,8 y 1,2 indican un ajuste óptimo, y entre 0,5 y 1,5 un ajuste aceptable ${ }^{34-36}$. Además, se realizó un análisis de umbrales por categorías para cada uno de los 5 factores del cuestionario. Finalmente, se realizó un mapa de ítems, que muestra la dispersión de puntuaciones para los ítems y para las personas, y un gráfico de la curva entre la puntuación directa y el valor de la variable latente (theta) medida (actitud hacia la prevención). Para estos análisis se utilizó el programa JMetrik ${ }^{36}$.

Para el análisis descriptivo de las variables se usó la media y desviación estándar o bien frecuencia y porcentaje, según el tipo de variable. Para el análisis inferencial de contraste de hipótesis se usaron test no paramétricos (Mann-Whitney y Kruskal-Wallis), ya que la puntuación APuP no se ajustaba a una distribución normal. El nivel de significación estadística se estableció en $p<0,05$. Para conocer el tamaño del efecto se utilizó la diferencia estandarizada de medias ( $d$ de Cohen), considerando $\mathrm{d}<0,5$ efecto pequeño; entre 0,5 y 0,8 , efecto moderado, y más de 0,8 , efecto fuerte. Para el análisis de datos utilizó el programa estadístico SPSS 21.0.

\section{Aspectos éticos}

Este estudio es parte de un proyecto de investigación mayor (proyecto SECOACBA; v. https://cuidsalud.com/portfolio/proyecto-secoacba/), que fue aprobado por el Comité de Ética de investigación biomédica de Jaén. Los participantes fueron informados del objetivo del estudio solicitando su aceptación, indicando que al completar y enviar los cuestionarios aceptaban participar. No se recogió ningún dato personal identificativo, por lo que se garantizó el anonimato en las respuestas.

\section{RESULTADOS}

Participaron en el estudio 228 estudiantes (la tasa de respuesta fue del $37,3 \%$ ), aunque tras codificación y depuración de los datos se eliminaron 40 cuestionarios incompletos, por lo que la muestra final fue de 188 cuestionarios. La tabla 1 muestra las características de la muestra de estudiantes.

\section{Análisis psicométrico del cuestionario APuP (versión española)}

El análisis del cuestionario APuP (versión española) con el modelo de Rasch para variables policotómicas resultó en una fiabilidad de 0,98 para los ítems y de 0,73 para las personas. Los índices de ajuste de los ítems fueron buenos, con todos los valores de WMS y UMS entre 0,5 y
Tabla 1. Características de estudiantes del Grado en Enfermería que participaron en el estudio $(\mathrm{N}=188)$

\begin{tabular}{|c|c|c|}
\hline Variable & & Frecuencia (\%) \\
\hline Edad [media, (DE)] & & $22(6)$ \\
\hline & Primero & $49(26,1 \%)$ \\
\hline & Segundo & $63(33,5 \%)$ \\
\hline Curso & Tercero & $43(22,9 \%)$ \\
\hline & Cuarto & $33(17,6 \%)$ \\
\hline Gónorn & Masculino & $33(17,6 \%)$ \\
\hline Genero & Femenino & $155(82,4 \%)$ \\
\hline Trabajo previo en el ámbito & No & $170(90,4 \%)$ \\
\hline de la salud & Sí & $18(9,6 \%)$ \\
\hline Realización de prácticas & No & $91(48,4 \%)$ \\
\hline clínicas & Sí & $97(51,6 \%)$ \\
\hline & Hospital & $40(41,2 \%)$ \\
\hline & Centro de salud & $2(2,1 \%)$ \\
\hline Lurar donde han realizado & Hospital + Centro de salud & $18(18,6 \%)$ \\
\hline las prácticas clínicas & $\begin{array}{l}\text { Hospital + Residencia de } \\
\text { mayores }\end{array}$ & $25(25,8 \%)$ \\
\hline & $\begin{array}{l}\text { Hospital + Centro de salud } \\
\text { + Residencia de mayores }\end{array}$ & $12(12,4 \%)$ \\
\hline Número de periodos & Uno & $41(42,7 \%)$ \\
\hline de prácticas realizados & Más de uno & $55(57,3 \%)$ \\
\hline Asistencia a jornadas/ & No & $140(74,5 \%)$ \\
\hline congresos sobre LPP último año & Sí & $48(25,5 \%)$ \\
\hline
\end{tabular}

DE: desviación estándar; LPP: lesiones por presión.

1,5 (tabla 2). Mediante el análisis Rasch también se midió el índice de dificultad de cada ítem (los valores más altos indican mayor dificultad de acuerdo en ese ítem y los valores negativos menor dificultad). Los dos ítems con menor grado de acuerdo son el 7 ("Una úlcera por presión casi nunca causa malestar a un paciente") y el 5 ("La prevención de las úlceras por presión no es tan importante"); ambos son ítems redactados en forma negativa por lo que hay que invertir su puntuación. A pesar del buen ajuste general del modelo a la población de estudiantes, en 3 de los 5 factores las categorías no funcionan de manera adecuada, ya que los valores de los umbrales no van ordenados de más negativo a más positivo en los factores 2,4 y 5 (tabla 3).

La consistencia interna global del cuestionario APuP (versión española) obtuvo un valor (alfa de Cronbach) de 0,74 [intervalo de confianza (IC) al 95\%: 0,68-0,79], aunque algunos de los factores tienen valores de alfa más bajos (tabla 3). No obstante, en este estudio se ha utilizado la puntuación global del cuestionario.

En el mapa de los ítems del cuestionario APuP (fig. 1) se muestra, en el lado izquierdo, la distribución de las puntuaciones obtenidas por las personas, indicando los valores superiores a 0 una actitud hacia la prevención más positiva y los inferiores a 0 una actitud menos positiva; y en el lado derecho, se muestra la distribución de los ítems según su actitud positiva, ordenados en el eje horizontal del 1 al 13. En el eje vertical se muestra el grado de acuerdo, indicando los valores más altos mayor grado de acuerdo. Los ítems 5, 7 y 11 están por encima de la habilidad de las personas, lo que indica que los estudiantes y las estudiantes muestran poco acuerdo con estos ítems. 
Tabla 2. Análisis Rasch del cuestionario APuP (versión en español) en estudiantes de enfermería

\begin{tabular}{|c|c|c|c|}
\hline \multirow{2}{*}{ Ítem } & \multirow{2}{*}{ Dificultad $^{\mathrm{a}}$} & \multicolumn{2}{|c|}{ Índices de ajuste } \\
\hline & & WMS & UMS \\
\hline 1. Tengo confianza en mi habilidad para prevenir las úlceras por presión & $-1,56$ & 1,02 & 1,00 \\
\hline 2. Tengo buena formación práctica para prevenir las úlceras por presión & $-2,15$ & 0,90 & 0,91 \\
\hline 3. La prevención de las úlceras por presión es demasiado difícil. Otros lo hacen mejor que yo & $-1,03$ & 1,07 & 1,07 \\
\hline 4. Se presta demasiada atención a la prevención de las úlceras por presión & $-0,25$ & 1,03 & 1,21 \\
\hline 5. La prevención de las úlceras por presión no es tan importante & 1,45 & 1,20 & 0,96 \\
\hline 6. La prevención de las úlceras por presión debería ser una prioridad & 0,53 & 0,84 & 0,88 \\
\hline 7. Una úlcera por presión casi nunca causa malestar a un paciente & 2,20 & 0,96 & 0,75 \\
\hline 8. El impacto económico de las úlceras por presión en los pacientes no se debería exagerar & $-0,65$ & 1,47 & 1,54 \\
\hline 9. El impacto económico de las úlceras por presión en la sociedad es alto & $-0,71$ & 1,07 & 1,11 \\
\hline 10. No soy responsable si se desarrolla una úlcera por presión en mis pacientes & 0,29 & 0,92 & 1,07 \\
\hline 11. Tengo un papel importante en la prevención de las úlceras por presión & 0,95 & 0,75 & 0,57 \\
\hline 12. Las úlceras por presión se pueden prevenir en pacientes con alto riesgo & 0,13 & 0,95 & 0,97 \\
\hline 13. Las úlceras por presión casi nunca son prevenibles & 0,82 & 0,97 & 0,88 \\
\hline
\end{tabular}

Valores mayores indican mayor dificultad de acuerdo.

UMS: Unweighted Mean Squared; WMS: Weighted Mean Squared.

Tabla 3. Cuestionario APuP: análisis de puntuaciones umbral por factores y consistencia interna

\begin{tabular}{|c|c|c|c|}
\hline Factor & Categoría & Umbrales & $\begin{array}{l}\text { Consistencia } \\
\text { interna alfa } \\
\text { (IC95\%) }\end{array}$ \\
\hline \multirow{4}{*}{$\begin{array}{l}\text { Factor 1. Competencia } \\
\text { personal para prevenir las } \\
\text { úlceras por presión }\end{array}$} & 0 & & \multirow{4}{*}{$0,72(0,65-0,79)$} \\
\hline & 1 & $-2,15$ & \\
\hline & 2 & 0,44 & \\
\hline & 3 & 1,71 & \\
\hline \multirow{4}{*}{$\begin{array}{l}\text { Factor 2. Prioridad de la } \\
\text { prevención de las úlceras } \\
\text { por presión }\end{array}$} & 0 & & \multirow{4}{*}{$0,36(0,19-0,51)$} \\
\hline & 1 & $-1,24$ & \\
\hline & 2 & 0,92 & \\
\hline & 3 & 0,32 & \\
\hline \multirow{4}{*}{$\begin{array}{l}\text { Factor 3. Impacto de las } \\
\text { úlceras por presión }\end{array}$} & 0 & & \multirow{4}{*}{$0,20(0-0,38)$} \\
\hline & 1 & $-1,63$ & \\
\hline & 2 & 0,34 & \\
\hline & 3 & 1,30 & \\
\hline \multirow{4}{*}{$\begin{array}{l}\text { Factor 4. Responsabilidad en } \\
\text { la prevención de las úlceras } \\
\text { por presión }\end{array}$} & 0 & & \multirow{4}{*}{$0,62(0,49-0,61)$} \\
\hline & 1 & $-0,96$ & \\
\hline & 2 & 1,14 & \\
\hline & 3 & $-0,18$ & \\
\hline \multirow{4}{*}{$\begin{array}{l}\text { Factor } 5 \text {. Confianza en la } \\
\text { eficacia de la prevención }\end{array}$} & 0 & & \multirow{4}{*}{$0,33(0,11-0,50)$} \\
\hline & 1 & $-2,17$ & \\
\hline & 2 & 1,28 & \\
\hline & 3 & 0,89 & \\
\hline
\end{tabular}

Valores mayores indican mayor dificultad de acuerdo.

UMS: Unweighted Mean Squared; WMS: Weighted Mean Squared.

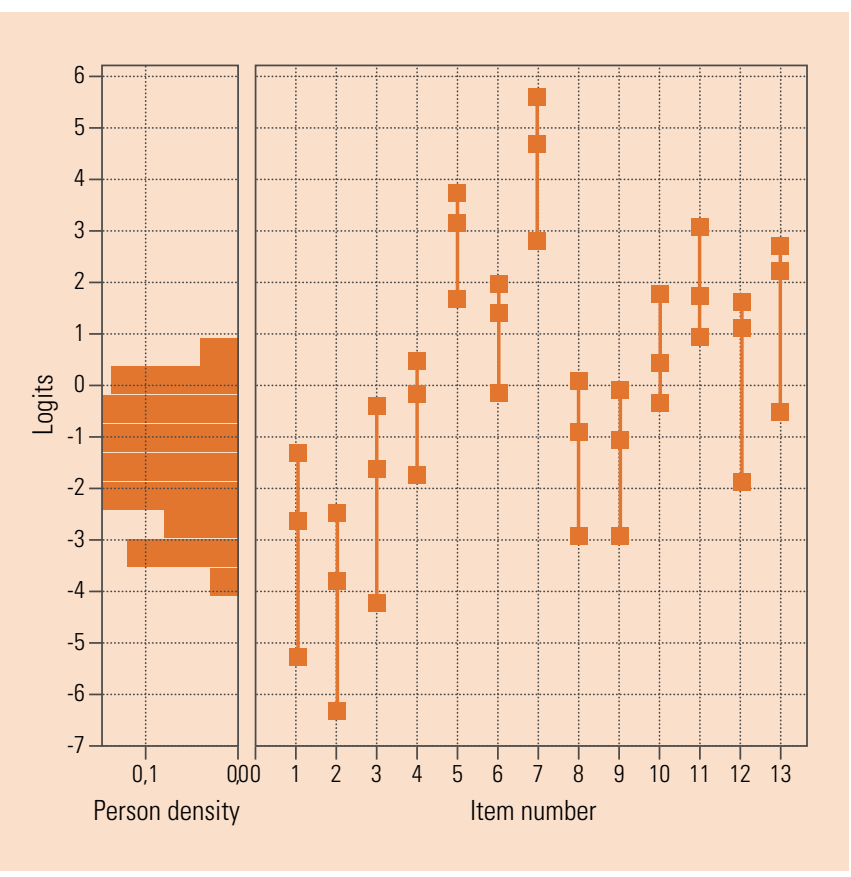

Figura 1. Mapa de ítems del cuestionario APuP (versión española). Fuente: elaboración propia.

Mediante el análisis de Rasch, es posible calcular el error de medición para cada ítem y calcular un valor real de la variable latente medida (denominado por la letra griega theta) (actitud hacia la prevención de LPP, en este caso). La figura 2 muestra la curva de ajuste entre las puntuaciones directas en el cuestionario y su correspondencia de valor verdadero (theta). Se puede observar en la parte inferior del gráfico que la relación no es lineal sino sigmoide, de forma que la disminución de 1 punto en la puntuación global supone una disminución mayor de 1 punto de la variable latente, es decir actitud menos positiva. 


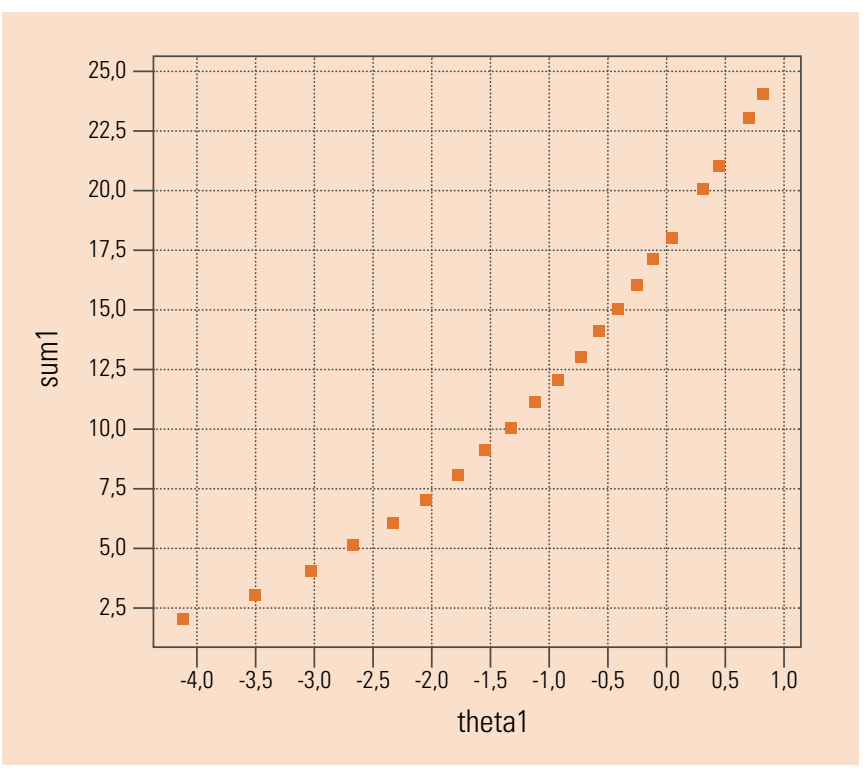

Figura 2. Gráfico de ajuste entre la puntuación directa y la variable latente (theta) medida mediante el cuestionario APuP (versión española).

Fuente: elaboración propia.

\section{Actitud hacia la prevención en estudiantes en el cuestionario APuP}

Tras la aplicación del cuestionario APuP (versión española) en la muestra de estudiantes de la Universidad de Jaén se obtuvo una puntuación media de 22,60 [desviación estándar (DE) 5,60; IC 95\% 21,82-23,43] sobre una puntuación máxima de 52 puntos; lo que es un $44,23 \%$ del máximo. En el cuestionario original, Beeckman estableció que una puntuación superior al $75 \%$ indicaba una actitud favorable ${ }^{22}$. La tabla 4 muestra las puntuaciones en cada uno de los ítems, ordenadas de mayor a menor. Los 3 ítems con mayor puntuación pertenecen al factor 1 (Competencias personales) y son "Tengo buena formación práctica para prevenir las úlceras por presión" (i2), "Tengo confianza en mi habilidad para prevenir las úlceras por presión" (i1) y "La prevención de las úlceras por presión es demasiado difícil. Otros lo hacen mejor que yo" (i3), con puntuación invertida. Por otro lado, los ítems con menor puntuación pertenecen al factor Prioridad: "La prevención de las úlceras por presión no es tan importante" (i5) y al factor Impacto "Una úlcera por presión casi nunca causa malestar a un paciente" (i7).

\section{Factores asociados a la actitud hacia la prevención de LPP}

La tabla 5 muestra los datos del análisis bivariante para comprobar qué variables están asociadas con la puntuación en el cuestionario APuP. Se halló que la puntuación de actitud no está asociada al sexo de los estudiantes $(p=0,235)$. Tampoco se halló asociación estadísticamente significativa con la asistencia a jornadas/congresos sobre LPP en el último año $(p=0,072)$.

Se encontró una asociación moderada con la realización de prácticas clínicas ( $p<0,0001 ; d=0,51)$; sin embargo, no tiene asociación con el número de periodos de prácticas realizados $(p=0,052)$. La puntuación de actitud en los estudiantes de ambos sexos que ya han realizado prácticas clínicas es más baja (actitud menos positiva) que los que no han realizado todavía prácticas. También se encontró una asociación estadísticamente significativa con el curso $(p<0,0001)$, de forma que los estudiantes y las estudiantes de los dos cursos superiores $\left(3 .^{\circ}\right.$ y $\left.4 .^{\circ}\right)$ tenían menores puntuaciones (es decir actitud menos positiva) que los de los dos primeros cursos $\left(1 .^{\circ}\right.$ y $\left.2 .^{\circ}\right)$, según se comprobó mediante el tamaño de efecto de las diferencias: $d$ de Cohen entre $1 .^{\circ}$ y $2 .^{\circ} d=0,19$ (efecto pequeño); entre $1 .^{\circ}$ y $3 .^{\circ} d=0,73$ (efecto moderado); entre $1 .^{\circ}$ y $4 .{ }^{\circ}$ $d=0,75$ (efecto moderado); entre $2^{\circ}$ y $3^{\circ} d=0,70$ (efecto moderado); entre $2^{\circ}$ y $4^{\circ} d=0,76$ (efecto moderado).

La puntuación de actitud (APuP) mostró una correlación moderada e inversa con la puntuación de conocimientos sobre prevención (cuestionario PIPK) Spearman $r=-0,46(p<0,0001)$, lo que indica que mayor conocimiento sobre prevención se asocia con una actitud menos positiva.

Tabla 4. Puntuaciones en los ítems del cuestionario APuP (versión española) en estudiantes de enfermería ( $N=188$ )

2. Tengo buena formación práctica para prevenir las úlceras por presión

1. Tengo confianza en mi habilidad para prevenir las úlceras por presión

3. La prevención de las úlceras por presión es demasiado difícil. Otros lo hacen mejor que yo

9. El impacto económico de las úlceras por presión en la sociedad es alto

8. El impacto económico de las úlceras por presión en los pacientes no se debería exagerar

4. Se presta demasiada atención a la prevención de las úlceras por presión

13. Las úlceras por presión casi nunca son prevenibles

10. No soy responsable si se desarrolla una úlcera por presión en mis pacientes 
Tabla 5. Asociación entre variables de formación y la puntuación en el cuestionario APuP en estudiantes de enfermería

\begin{tabular}{|c|c|c|c|}
\hline \multicolumn{2}{|l|}{ Variable } & $\begin{array}{c}\text { Puntuación } \\
\text { APuP } \\
\text { Media (DE) }\end{array}$ & $\begin{array}{l}\text { Significación } \\
\text { estadística }^{\mathrm{a}}\end{array}$ \\
\hline \multirow{2}{*}{ Género } & Hombre & $23,33(6,04)$ & \multirow{2}{*}{$p=0,235$} \\
\hline & Mujer & $22,47(5,51)$ & \\
\hline \multirow{2}{*}{$\begin{array}{l}\text { Realización de prácticas } \\
\text { clínicas }\end{array}$} & No & $24,07(6,32)$ & \multirow{2}{*}{$p<0,0001$} \\
\hline & Sí & $21,27(4,44)$ & \\
\hline \multirow{2}{*}{$\begin{array}{l}\text { Número de periodos de } \\
\text { prácticas clínicas }\end{array}$} & Uno & $22,37(4,28)$ & \multirow{2}{*}{$p=0,052$} \\
\hline & Más de uno & $20,31(4,31)$ & \\
\hline \multirow{2}{*}{$\begin{array}{l}\text { Asistencia a jornadas o } \\
\text { congresos sobre LPP último año }\end{array}$} & No & $22,82(6,13)$ & \multirow{2}{*}{$p=0,072$} \\
\hline & Sí & $22,04(3,63)$ & \\
\hline \multirow{4}{*}{ Curso en que está matriculado } & $1 .{ }^{\circ}$ & $24,76(7,21)$ & \multirow{4}{*}{$p<0,0001$} \\
\hline & $2 .^{\circ}$ & $23,53(4,77)$ & \\
\hline & $3 .^{\circ}$ & $20,49(4,91)$ & \\
\hline & $4 .^{\circ}$ & $20,31(2,62)$ & \\
\hline
\end{tabular}

${ }^{a}$ Test Mann-Whitney, excepto para curso matriculado (test Kruskal-Wallis).

\section{У DISCUSIÓN}

Esta investigación ha mostrado que la versión espańola del cuestionario APuP tiene adecuadas propiedades psicométricas de ajuste y fiabilidad, para su uso en estudiantes de enfermería. Permite medir la actitud más o menos positiva hacia la prevención de las LPP e identificar en qué factores o áreas esta actitud es más favorable. Por otro lado, se halló que en la muestra de estudiantes del Grado en Enfermería de la Universidad de Jaén la puntuación de actitud no llega al nivel para ser considerada como positiva.

La versión espańola del cuestionario APuP fue desarrollada y validada en profesionales de enfermería y técnicos de cuidados auxiliares de enfermería (TCAE) en 4 hospitales de Jaén, a partir del instrumento original en inglés ${ }^{22,30}$. En esta población de profesionales mostró adecuada fiabilidad y validez y un buen ajuste al modelo de 5 factores. La utilización de la Teoría de Respuesta al Ítem mediante el análisis de Rasch que se ha empleado en este estudio aporta un valor ańadido a la investigación, ya que permite una validación más robusta del cuestionario, el análisis separado de los efectos de los ítems y de las personas, y la estimación de valor de la variable latente medida. Este método de análisis de cuestionarios es recomendado para instrumentos sobre rasgos personales y dominios cognitivos que no son directamente observables, como son los conocimientos o las actitudes ${ }^{36}$, aunque aún es escasamente utilizado en estudios en el área de enfermería en español ${ }^{37}$.

Todos los ítems del cuestionario ofrecen un buen ajuste en el modelo y unos índices de dificultad adecuados, aunque en esta población de estudiantes se identificaron 3 ítems con bajo nivel de acuerdo. Es posible que estos ítems se refieran a aspectos de la prevención de las LPP que los estudiantes de ambos sexos todavía no tienen claros, o bien que no hayan sido bien entendidos en su redacción. En la literatura científica no hemos identificado ningún estudio sobre cuestionarios en estudiantes que ofrezca un conjunto amplio de valores de propiedades psicométricas, algunos solo indican la consistencia interna, como la investigación en estudiantes de enfermería italianos (alfa $=0,88)^{25}$, pero lo más frecuente es que no se mencionen datos psicométricos del cuestio- nario utilizado ${ }^{24,26}$. Actualmente, la inclusión de datos sobre las propiedades psicométricas de los instrumentos tipo cuestionario usados en las investigaciones se considera un punto de especial importancia, ya que contribuye a dar seguridad en la fiabilidad y consistencia de los datos presentados. Los estudios basados en instrumentos, cuyas propiedades de fiabilidad y validez no se conocen, deben interpretarse con mucha cautela debido al posible error en la medición.

Esta investigación ha puesto de manifiesto que entre los estudiantes y las estudiantes de enfermería de la Universidad de Jaén la actitud hacia la prevención de las LPP no es positiva, puesto que no alcanzó el 50\% de la puntuación máxima con el cuestionario APuP (el autor del cuestionario $\mathrm{APuP}$ propuso un $75 \%$ del máximo como actitud positiva ${ }^{22}$ ). La puntuación de actitud en la muestra de estudiantes de Jaén es inferior al descrito en otras investigaciones en estudiantes de enfermería italianos $(76,7 \%)^{25}$, irlandeses $(83,3 \%)^{24}$, australianos $(78 \%)^{26}$ o turcos $(77 \%)^{8}$, aunque los cuestionarios usados en la medición no fueron los mismos en todos los casos. Este hallazgo de actitud poco favorable a la prevención de las LPP en estudiantes espańoles no había sido descrito anteriormente, por lo que debería ser analizado de forma más profunda, estudiando un número más grande de facultades de enfermería para tener una muestra representativa. En cualquier caso, indica que el desarrollo de actitudes positivas hacia las LPP y su prevención precisa ser considerado de forma expresa en los planes formativos y no solo el enfoque en los contenidos para desarrollar conocimientos.

El análisis detallado de las respuestas de los estudiantes de ambos sexos permitió identificar que los ítems con mayor puntuación fueron los relacionados con la competencia personal para prevenir las LPP y los que menos fueron los relacionados con el impacto y la prioridad de la prevención de las LPP. Esto indica que las estudiantes y los estudiantes de enfermería de la Universidad de Jaén perciben que tienen habilidad para proveer un adecuado cuidado preventivo para evitar las LPP, sin embargo, consideran bajo el impacto que estas lesiones relacionadas con la dependencia tienen sobre la sociedad y los pacientes. Esta situación se podría resumir como "yo sé cómo prevenir estas lesiones, pero no hace falta porque no son un problema importante" y evidencia una actitud que puede limitar mucho que las medidas preventivas se pongan en práctica en los entornos asistenciales. En este sentido, estos resultados discrepan de los mencionados en otros estudios, en los que el factor impacto tiene una actitud más positiva y el factor competencia personal tiene menos ${ }^{8,26}$. También el estudio de Florin et al. ${ }^{23}$ en Suecia identificó el factor competencia personal para prevenir las LPP como el menos puntuado.

Respecto a los factores relacionados con la actitud hacia la prevención de las LPP, en este estudio no encontramos asociación con el sexo, al contrario que Usher et al. ${ }^{26}$ y Kisacik et al. ${ }^{8}$, quienes encontraron que las estudiantes tenían actitudes más positivas hacia la prevención de LPP que los estudiantes. Tampoco encontramos una asociación significativa entre la actitud y la asistencia a jornadas o congresos sobre LPP, coincidiendo con los resultados encontrados por Cullen Gill et al. ${ }^{24}$ en estudiantes de enfermería irlandeses. En cambio, otro estudio realizado en Turquía sí describe esta asociación, de forma que aquellos estudiantes que habían realizado cursos relacionados con las UPP mostraban una actitud más positiva que los que no lo habían realizado, encontrando diferencias significativas $(77,3 \% \text { versus } 74,4 \% ; p=0,033)^{8}$.

En la muestra de estudiantes de Jaén encontramos una asociación moderada entre la actitud y la realización de prácticas clínicas, pero en sentido contrario al inicialmente esperado, ya que la actitud es menos positiva en estudiantes que han realizado prácticas clínicas frente a los que aún no las han realizado. Este resultado es opuesto al obtenido en otras investigaciones que describen una correlación positiva (aunque débil) entre el número 
de días de práctica clínica por semana, la duración y el número de pacientes que tenían $\mathrm{LPP}^{8}$. En nuestro estudio tampoco se encontró asociación significativa entre la puntuación de actitud y el número de unidades en las que habían realizado prácticas, al contrario que en el estudio realizado con los estudiantes de enfermería italianos y australianos ${ }^{25,26}$. Esta asociación inversa entre la actitud hacia la prevención de las LPP y las prácticas clínicas constituye un hallazgo novedoso que no había sido descrito previamente. Puesto que este estudio se hizo en una única universidad espańola, sería necesario comprobarlo en un número mayor de centros para valorar si se trata de una asociación real o un efecto del azar. Si se trata de una verdadera asociación, sería necesario establecer sus posibles causas. Como hipótesis proponemos que el contacto de las estudiantes y los estudiantes con los entornos clínicos reales los expone a una subestimación de la importancia del problema de las LPP, que con frecuencia es considerado un problema menor o una situación "inevitable".

En nuestro estudio también encontramos una asociación entre la actitud hacia la prevención y el curso del Grado en Enfermería en que están matriculados, de forma que estudiantes de cursos superiores $\left(3 .^{\circ}\right.$ y $\left.4 .^{\circ}\right)$ tienen una actitud menos positiva que estudiantes de los primeros cursos. Nuevamente este resultado discrepa de resultados publicados previamente por otros autores que muestran una asociación positiva, es decir mejor actitud en los cursos superiores ${ }^{8,25,26}$. Puesto que ambos factores (prácticas clínicas y cursos superiores) van vinculados, ya que todos los estudiantes realizan más periodos de prácticas en los cursos superiores, es posible que el efecto sobre la actitud sea conjunto o bien predomine uno de los dos factores, siendo otra cuestión que queda pendiente de resolver. Es posible que tal como indican Kielo et al..$^{38}$, los estudiantes pueden ser conscientes de la importancia de la prevención de las LPP, pero cuando realizan las prácticas clínicas les resulta complejo y disminuye su motivación y, por consiguiente, su actitud hacia la prevención de las LPP.

Finalmente, es destacable la existencia de una correlación inversa entre la puntuación de conocimientos sobre prevención y la puntuación de actitud hacia la prevención, lo que coincide con la conclusión de Cullen Gill et al. ${ }^{24}$ sobre que un mayor nivel de conocimiento no equivale a una actitud más positiva hacia la prevención de LPP. Este hallazgo resulta de enorme importancia, ya que con frecuencia los planes de estudios del Grado en Enfermería se centran solo en la adquisición de conocimientos sobre la prevención, pero no se aborda de forma expresa y planificada el desarrollo de una actitud positiva en los estudiantes.
Según los resultados de este estudio y los publicados por otros autores, parece que sería necesario realizar más investigaciones que midan tanto los conocimientos como la actitud hacia la prevención de LPP de los estudiantes de enfermería en los diferentes cursos del grado, con el fin de tener una perspectiva más amplia y real de la situación. En este sentido, tanto el cuestionario de conocimientos PIPK $^{18}$ como el de actitud $\mathrm{APuP}$, ambos en versión española, son instrumentos útiles para su uso en estudiantes. Resulta especialmente importante comprobar algunos de los hallazgos de este estudio, como la correlación inversa entre conocimientos y actitud, y el efecto de las prácticas clínicas y el curso sobre la actitud de las estudiantes y los estudiantes.

Este estudio presenta algunas limitaciones que deben tenerse en cuenta. Puesto que el muestreo fue por conveniencia y en un único centro, la extrapolación y generalización de los resultados al resto de universidades españolas debe hacerse con mucha prudencia, y haría falta replicar el estudio en un número mayor de centros. Además, la tasa de respuesta relativamente baja $(37,3 \%)$ podría llevar a una sobreestimación de los resultados, ya que las estudiantes y los estudiantes más motivados o interesados pueden ser los que más hayan respondido el formulario.

\section{$\searrow$ CONCLUSIONES}

El cuestionario APuP (versión española) presenta buenos indicadores psicométricos en estudiantes de enfermería, por lo que resulta un instrumento útil. En la muestra de estudiantes del Grado en Enfermería de Jaén, la actitud hacia la prevención no es positiva, sobre todo debido a la poca valoración de la prioridad y el impacto de las LPP. Se ha puesto en evidencia que los estudiantes de cursos superiores y quienes han realizado prácticas clínicas en centros sanitarios tienen una actitud menos positiva hacia la prevención respecto a estudiantes de primer curso

\section{Conflicto de intereses}

Los autores declaran no tener ningún conflicto de intereses.

\section{Agradecimientos}

Agradecemos al profesorado y estudiantes de la Facultad de Ciencias de la Salud de la Universidad de Jaén su colaboración para el desarrollo de este estudio de investigación.

\section{У BIBLIOGRAFÍA}

1. García-Fernández FP, Soldevilla Agreda JJ, Verdú J, Pancorbo-Hidalgo PL. A new theoretical model for the development of pressure ulcers and other dependence-related lesions. J Nurs Scholarsh. 2014:46(1):28-38

2. National Pressure Ulcer Advisory Panel (NPUAP). Change in terminology from pressure ulcer to pressure injury and updates the stages of pressure injury. Disponible en: https://npiap.com/ page/PressurelnjuryStages

3. Pancorbo-Hidalgo PL, García-Fernández FP, Pérez-López C, Soldevilla-Ágreda JJ. Prevalencia de lesiones por presión y otras lesiones cutáneas relacionadas con la dependencia en población adulta en hospitales españoles: resultados del $5^{\circ}$ Estudio Nacional de 2017. Gerokomos. 2019;30:76-86.

4. Pancorbo-Hidalgo PL, Torra-Bou JE, García-Fernández FP, Soldevilla-Ágreda JJ. Prevalence of pressure injuries and other dependence-related skin lesions among pediatrics patients in hospitals in Spain. EWMA J. 2018;19:29-37.

5. Demarré L, Vanderwee K, Defloor T, Verhaeghe S, Schoonhoven L, Beeckman D. Pressure ulcers: knowledge and attitude of nurses and nursing assistants in Belgian nursing homes. J Clin Nurs. 2011;21(9-10):1425-34.
6. Jackson D, Hutchinson M, Barnason S, Li W, Mannix J, Neville S, et al. Towards international consensus on patient harm: perspectives on pressure injury policy. J Nurs Manag. 2016;24(7):902-14.

7. Jackson D, Durrant L, Bishop E, Walthall H, Betteridge R, Gardner $\mathrm{S}$, et al. Health service provision and the use of pressure redistributing devices: mixed methods study of community dwelling individuals with pressure injuries. Contemp Nurse. 2017:53(3):378-89.

8. Kısacık ÖG, Sönmez M. Pressure ulcers prevention: Turkish nursing students' knowledge and attitudes and influencing factors. J Tissue Viability. 2020;29(1):24-31.

9. Blanco-Zapata RM, López-García E, Quesada-Ramos C. Guía de Actuación para la Prevención y Cuidados de las Úllceras por Presión. Osakidetza; Octubre 2017. Disponible en: https://bit.ly/32KMuZa

10. Beeckman D, Vanderwee K, Demarre L, Paquay L, Van Hecke A Defloor T. Pressure ulcer prevention: development and psychometric validation of a knowledge assessment instrument. Int $\mathrm{J}$ Nurs Stud. 2010:47(4):399-410.

11. Garrigues LJ, Cartwright JC, Bliss DZ. Attitudes of nursing students about pressure injury prevention. J Wound Ostomy Continence Nurs. 2017:44(2):123-8.
12. Mallah Z, Nassar N, Kurdahi Badr L. The effectiveness of a pressure ulcer intervention program on the prevalence of hospital acquired pressure ulcers: controlled before and after study. Appl Nurs Res. 2015;28(2):106-13

13. Moore Z. Pressure ulcer prevention: nurses' knowledge, attitudes and behavior. J Wound Care. 2004;13(8):330-4.

14. López-Franco MD, Pancorbo-Hidalgo PL. Instrumentos de medición de los conocimientos sobre prevención de úlceras por presión: revisión de la literatura. Gerokomos. 2019;30(2):98-106.

15. Pieper B, Mott M. Nurses' knowledge of pressure ulcer prevention, staging, and description. Adv. Wound Care. 1995;8:34-40.

16. Pieper B, Zulkowski K. The Pieper-Zulkowski pressure ulcer knowledge test. Adv. Skin Wound Care. 2014;27:413-9.

17. Manderlier B, Van Damme N, Vanderwee K, Verhaeghe S, Van Hecke A, Beeckman D. Development and psychometric validation of PUKAT 20, a knowledge assessment tool for pressure ulcer prevention. Int. Wound J. 2017:14:1041-51.

18. López-Franco MD, Parra-Anguita L, Comino-Sanz IM, Pancorbo-Hidalgo PL. Development and Psychometric Properties of the Pressure Injury Prevention Knowledge Questionnaire in Spanish Nurses. Int J Environ Res Public Health. 2020;17(9):3063. 
19. López-Franco MD, Parra-Anguita L, Pancorbo-Hidalgo PL. Instrumentos de medición de las actitudes y las barreras para la prevención de lesiones por presión: revisión de la bibliografía. Gerokomos. 2019;30(3):217-25

20. Moore Z, Price P. Nurses' attitudes, behaviours and perceived barriers towards pressure ulcer prevention. J Clin Nurs. 2004;13:942-51.

21. Beeckman D, Defloor T, Demarre L, Van Hecke A, Vanderwee K. Pressure ulcers: development and psychometric evaluation of the attitude towards pressure ulcer prevention instrument (APuP). Int J Nurs Stud. 2010;47(11):1432-41.

22. Beeckman D, Defloor T, Schoonhoven L, Vanderwee K. Knowledge and attitudes of nurses on pressure ulcer prevention: a cross-sectional multicenter study in Belgian hospitals. Worldviews Evid Based Nurs. 2011;8(3):166-76.

23. Florin J, Baath C, Gunningberg L, Martensson G. Attitudes towards pressure ulcer prevention: a psychometric evaluation of the Swedish version of the APuP instrument. Int Wound J. 2016;13(5):655-62.

24. Cullen Gill E, Moore Z. An exploration of fourth-year undergraduate nurses' knowledge of and attitude towards pressure ulcer prevention. J Wound Care. 2013;22(11):618-27.

25. Simonetti V, Comparcini D, Flacco ME, Di Giovanni P, Cicolini G. Nursing students' knowledge and attitude on pressure ulcer prevention evidence based guidelines: a multicenter cross sectional study. Nurse Educ Today. 2015;35(4):573-9.

26. Usher K, Woods C, Brown J, Power T, Lea J, Hutchinson M, et al Australian nursing students' knowledge and attitudes towards pressure injury prevention: a cross-sectional study. Int J Nurs Stud. 2018;81:14-20.

27. Gunningberg L, Mårtensson G, Mamhidir AG, Florin J, Muntlin Athlin $\AA$, Bååth C. Pressure ulcer knowledge of registered nurses, assistant nurses and student nurses: a descriptive, comparative multicentre study in Sweden. Int. Wound J. 2013;12(4):462-8.

28. Rafiei H, Mehralian H, Abdar M, Madadkar T. Pressure ulcers: how much do nursing students really know? Br. J. Nurs. 2015;24(6):S12-7.

29. Pérez-López C, López-Franco MD, Comino-Sanz IM, Pancorbo-Hidalgo PL. Validación del cuestionario de conocimientos sobre prevención de lesiones por presión en estudiantes de Enfermería: Análisis Rasch. Enferm Clin. 2020; en prensa.

30. López-Franco MD. La prevención de lesiones por presión: conocimientos, actitudes y barreras de los profesionales de enfermería [tesis doctoral]. Jaén: Universidad de Jaén; 2020.

31. Embretson SE, Reise SP. Item response theory for psychologists. 1. a ed. Mahwah: Psychology Press; 2013.
32. Rasch G. Probabilistic models for some intelligence and attainment tests (Copenhagen, Danish Institute for Educational Research), expanded edition (1980) with foreword and afterword by B.D. Wright. Chicago: The University of Chicago Press; 1981

33. Andrich DA. Rating formulation for ordered response categories. Psychometrika. 1978:43:561-73.

34. Linacre JM. User's guide to Winstep. Ministeps Rasch-Mode Computer Programs: Program Manual.3.73.0 [Internet]. 2018 [acceso 23 julio 2020]. Disponible en: http://www.winsteps. com/winman/copyright.htm

35. Campo-Arias A, Oviedo HC. Propiedades Psicométricas de una Escala: la Consistencia Interna. Rev Salud Pública. 2008;10(5): 831-9.

36. Meyer JP. Applied measurement with jMetrik. 1. ${ }^{\text {a }}$ ed. New York: Routledge; 2014.

37. Álvarez-García C, Álvarez-Nieto C, Pancorbo-Hidalgo PL, Sanz-Martos S, López-Medina IM. Student nurses' knowledge and skills of children's environmental health: Instrument development and psychometric analysis using item response theory. Nurse Educ Today. 2018:69:113-9.

38. Kielo E, Salminen L, Stolt M. Graduating student nurses' and student podiatrists' wound care competence - An integrative literature review. Nurse Educ Pract. 2018;29:1-7. 